\title{
THE RELATION BETWEEN MASS LOSS AND LUMINOSITY FOR Be STARS
}

\author{
L.B.F.M. Waters, H.J.G.L.M. Lamers, and J. Coté \\ SRON Laboratory for Space Research \\ Beneluxlaan 21 \\ 3527 HS Utrecht, Netherlands
}

\begin{abstract}
The mass loss rates of a large sample of Be stars derived from the UV and the IR are compared. The IR rates were derived using a simple equatorial disc model, and are typically a factor 100 larger than those derived from the UV. In terms of mass fluxes, the mass flux in the polar regions (derived from the UV observations) is about a factor $10^{3}$ smaller than the mass flux in the equatorial regions. The dependence of $\dot{M}_{I R}$ and $\dot{M}_{U V}$ on stellar luminosity is studied. It is shown that $\dot{\mathrm{M}}_{\mathrm{IR}}$ depends weaker on $\mathrm{L}$ than $\dot{\mathrm{M}}_{\mathrm{UV}}$. This suggests that two different mechanisms are responsible for the mass flux in polar and equatorial regions.
\end{abstract}

\section{INTRODUCTION}

The study of mass loss rates of Be stars in different wavelength regions shows an extremely discrepant picture. The rates derived from asymmetric UV resonance lines (e.g. Snow, 1981) are of the order of $10^{-10} \mathrm{M}_{\odot} / \mathrm{yr}$, whereas those derived from $\mathrm{H} \alpha$ line profiles and the IR excess (e.g. Poeckert and Marlborough, 1979) are typically $10^{-8} \mathrm{M}_{\odot} / \mathrm{yr}$. The wind characteristics derived from UV and IR observations are also very different. The UV observations suggest a high-velocity, low density wind, while the $\mathrm{H} \alpha$ and IR observations indicate a low-velocity, high-density wind. Obviously, the winds of Be stars are not homogeneous. The geometry of the winds is still a matter of debate. Thomas and Doazan (1982) suggest that the wind is spherically symmetric and is decelerated after initial acceleration. Alternately, the winds of Be stars may be disc-like (Struve, 1931; Poeckert and Marlborough, 1978) with the $\mathrm{H} \alpha$ and IR radiation originating from the high-density, low-velocity disc, and the UV resonance lines from the low-density, high velocity regions above and below the disc (the polar regions).

\section{THE MASS LOSS - LUMINOSITY DIAGRAM}

In Fig. 1, we plot the mass loss rates derived from IRAS observations by Waters et al. (1986) (circles and plusses), as well as those derived by Snow (1981) from UV observations for the stars in common (triangles), versus luminosity. The values for $\dot{M}_{I R}$ were derived using a simple pole-on disc model (Waters, 1986), i.e. taking into account the asymmetry of the stellar wind. The inferred mass loss rates are not affected strongly by the pole-on assumption unless the disc is viewed edge-on. The IR excess as a function of wavelength 


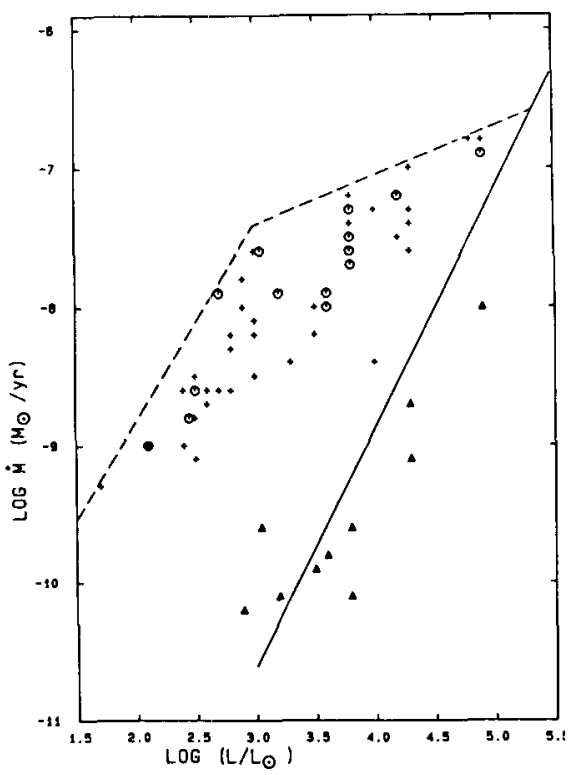

Figure 1: $\dot{\mathrm{M}}$ - L diagram for Be stars. Circles and plusses are $\dot{\mathrm{M}}_{\mathrm{IR}}$, triangles are $\dot{\mathrm{M}}_{\mathrm{UV}}$. The solid line represents the $\dot{M}$ - L relation for 0 stars. The dashed line is the upper limit to $\dot{\mathrm{M}}_{\mathrm{IR}}$.

yields the density structure in the equatorial regions, $\rho(\mathbf{r}) \sim \mathbf{r}^{-\mathbf{n}}$. In almost all cases $2<\mathrm{n}<3.5$ was found (Waters et al., 1986). Assuming mass continuity, this implies that material is flowing away at constant velocity or is very gradually accelerated outwards. Using the mass continuity equation and adopting a radial outflow velocity of $5 \mathrm{~km} / \mathrm{s}$ at the photosphere, the IR mass loss rates are derived. Three important conclusions can be drawn from this figure:

i. $\dot{M}_{U V}$ follows approximately the relation between $\dot{M}$ and L derived for the 0-type stars by Garmany et al. (1981). This relation is indicated by the solid line in Fig. 1. This was already noted by Snow (1982).

ii. $\dot{M}_{I R}$ is always much larger than $\dot{M}_{U V}$, typically a factor 100 . Furthermore, $\dot{M}_{I R}$ depends weaker on $L$ than $\dot{M}_{U V}$.

iii. An upper limit to $\dot{\mathrm{M}}_{\mathrm{IR}}$ as a function of $\mathrm{L}$ exists (dashed line).

\section{CONCLUSIONS}

It is clear that the wind characteristics of Be star winds derived from the UV and the IR are very different. The different dependence of $\dot{M}_{U V}$ and $\dot{M}_{I R}$ on L suggests that the basic mechanisms governing the mass loss in polar regions (the UV observations) and in equatorial regions (the IR and $\mathrm{H} \alpha$ observations) are different. The high-velocity, low-density character of the polar regions, combined with the strong dependence on luminosity suggests that the mechanism responsible for the mass loss in the polar regions is similar to that of the 0-type stars, i.e. radiation pressure. The low-velocity, high-density equatorial regions 
with a mass flux about $10^{3}$ times the polar mass flux do not show the same dependence on $\mathrm{L}$. This suggests that some other mechanism, the "Be mechanism", is responsible for the mass loss in the equatorial regions. A detailed analysis of the IRAS observations of $101 \mathrm{Be}$ stars will be published in Astronomy and Astrophysics.

\section{ACKNOWLEDGEMENTS}

LBFMW acknowledges financial support from the Netherlands Organization for Pure Scientific Research ZWO.

\section{REFERENCES}

Doazan, V., Thomas, R.N.: 1982, in: "B stars with and without Emission lines", NASA SP-456.

Garmany, C.D., Olson, G.L., Conti, P.S., Van Steenberg, M.E.: 1981, Astrophys. J., 250, 660.

Poeckert, R., Marlborough, J.M.: 1978, Astropys. J. Suppl., 38, 229.

Poeckert, R., Marlborough, J.M.: 1979, Astrophys. J., 233, 259.

Snow, T.P.: 1981, Astrophys. J., 251, 139.

Snow, T.P.: 1982, Astrophys. J. Suppl., 253, L39.

Struve, O.: 1931, Astrophys. J., 73, 94.

Waters, L.B.F.M.: 1986, Astron. Astrophys., 162, 121.

Waters, L.B.F.M., Coté, J., Lamers, H.J.G.L.M.: 1986 (in preparation). 


\section{DISCUSSION FOLLOWING WATERS}

Buscombe:

Is there any separation of your data according to stars which are members of binary systems?

Waters:

No there isn't. I assumed a Be star to be a spectroscopic binary only if there are published orbital elements of convincing quality; so according to this conservative view I found only two binary systems in my sample.

Lamers:

The only information about the velocity in the disks of the Be stars must come from profile studies. From the study of the emission profiles of $\gamma$ Cas by Poeckert and Marlborough, and by Chalabaev and Maillard it is suggested that $\mathrm{v}$ is about $2 \mathrm{kms}^{-1}$ and $20 \mathrm{kms}^{-1}$ respectively at $R_{*}$. So, we used a mean value of $5 \mathrm{kms}^{-1}$. Also the profiles of shell lines in shell stars show violet absorption wings, with $\mathrm{v}<50 \mathrm{kms}^{-1}$ (Oegerle and Polidan, 1984), again indicating that there is indeed outflow in the disks.

Henrichs:

Regarding your assumed expansion velocity of $5 \mathrm{kms}^{-1}$, wouldn't you at least expect to have the thermal speed, which is certainly higher? What consequences does a different assumed velocity have on your derived mass loss rates?

Waters:

The radial outflow velocity of $5 \mathrm{kms}^{-1}$ is very uncertain. The model for $\gamma$ Cas published by Poeckert and Marlborough adopts an outflow velocity of $8 \mathrm{~km} / \mathrm{s}$, and in the case of $\phi$ Per of $2 \mathrm{kms}^{-1}$, i.e. very subsonic. The mass loss rates are proportional to the adopted outflow velocity at the photosphere.

Henrichs:

In Snow's work a fixed ionization fraction was assumed simply because there is nothing better available. May I therefore suggest that you recognize the very substantial uncertainties caused by this procedure when you compare them with your mass-loss rates?

Waters:

I agree.

Underhill:

Once again I would like to know how your group can justify your statement that flow at your adopted velocity at the place you assume it occurs leads to actual loss (escape) of material from the star.

Waters:

The velocities in the equatorial regions (assuming there is outflow) are of the order of 10 to $50 \mathrm{kms}^{-1}$, i.e. less than escape velocity at a few stellar radii. This does not necessarily imply that the material cannot escape from the star. The escape velocity decreases with increasing distance, so even a small velocity will eventually exceed the escape velocity. For instance, the outflow velocities in the wind of the hypergiant $\mathrm{P}$ Cygni at $1<r<4 R_{*}$ do not exceed the local escape velocity, yet there is actual loss of material from the star. 
Dachs:

In support of Dr. Underhill's comment, I should like to point out that infrared spectral energy distributions of Be star can also be fitted by models of free-free + bound-free emission assuming emission from a stationary rotating disk, without requiring expansion. 